\title{
Estudio Exploratorio de Higiene Industrial en Ambientes de Trabajo Donde Se Producen o Utilizan Nanopartículas
}

\section{EXPLORATORY STUDY OF INDUSTRIAL HYGIENE IN WORK AREAS WHERE NANOPARTICLES ARE PRODUCED OR USED}

Rómulo Zúñiga Rojas', Ximena Blamey Benavides², Edgar Mosquera ${ }^{3}$, Leonardo Ahumada Bolton ${ }^{4}$

1. Ingeniero Civil Quimico, Subgerencia de Especialidades Técnicas, Asociación Chilena de Seguridad. Ramón Carnicer 163, Providencia, Santiago, Chile.

2. Ingeniero Civil Químico, MBA, Experto Profesional en Prevención de Riesgos, Consultor en Sistemas de Gestión, Santiago, Chile.

3. Doctor en Fisica-Química, Laboratorio de Materiales a Nanoescala, Departamento de Ciencia de los Materiales, Universidad de Chile, Tupper 2069, piso 2 y 3, Santiago, Chile.

4. Ingeniero Civil en Materiales, Experto Profesional en Prevención de Riesgos, Departamento Seguridad Industrial \&t Medio Ambiente, Asociación Chilena de Seguridad. Ramón Carnicer 163, Santiago, Chile.

\section{RESUMEN}

Se realizó un estudio exploratorio con los objetivos de identificar empresas que produzcan o manipulen nanopartículas en Chile, evaluar los riesgos para la salud de estas actividades y entregar recomendaciones para su control. Se encontró que la nanotecnología está en una etapa incipiente, con un reducido número de aplicaciones en procesos industriales, concentrándose el desarrollo y aplicación principalmente en centros de investigación de las universidades, donde los investigadores y estudiantes son el principal grupo de potenciales expuestos. Para evaluar la exposición en las empresas e instituciones identificadas, se utilizó un método cualitativo simplificado y un método cuantitativo que se basa en las técnicas tradicionales de higiene ocupacional complementadas con la caracterización de las nanopartículas mediante microscopía electrónica. Se evalúa la exposición a nanopartículas de cobre, carbonato de calcio y partículas ultrafinas de humos de soldadura. La metodología de evaluación cualitativa, si bien requiere algún grado de acondicionamiento, demostró ser una herramienta particularmente útil para definir sistemas de protección en el caso de las nanopartículas, cuya toxicidad es incierta y de las cuales no se tienen establecidas técnicas de medición ni límites permisibles. Los métodos de medición tradicionales de la higiene industrial no entregan la información suficiente para evaluar la exposición, debiendo ser complementados con microscopía electrónica para caracterizar el tamaño y composición química, parámetros claves en la toxicidad de las nanopartículas. El trabajo realizado entrega una base de información y conocimiento a nivel exploratorio, que se estima útil para evaluar el riesgo de exposición en aplicaciones que pudieran surgir en el contexto actual del desarrollo de la nanotecnología en nuestro país.

(Zúñiga R, Blamey X, Mosquera E, Ahumada L, 2013. Estudio Exploratorio de Higiene Industrial en Ambientes de Trabajo Donde Se Producen o Utilizan Nanopartículas. Cienc Trab. Sep-Dic; 15 [48]: 124-130).

Palabras clave: NANOTECNOLOGÍA, NANOPARTÍCULAS, NANOMATERIALES, HIGIENE INDUSTRIAL, EVALUACIÓN CUALITATIVA, EVALUACIÓN CUANTITATIVA, TOXICIDAD, PARTÍCULAS ULTRAFINAS.

\section{ABSTRACT}

An exploratory research was developed in order to identify companies which produce or handle nanoparticles in Chile, evaluate the risks for health from these activities and give suggestions for controlling them. It was found that nanotechnology is in an incipient stage, with a reduced numbers of applications in industrial processes, focusing the development and application mainly on research centers in Universities, where researchers and students are the main exposed potential groups. To evaluate the exposure in identified companies and institutions, it was used a qualitative simplified method and a quantitative method which is based in traditional technique of occupational hygiene complemented by the characterization of nanoparticles with electronic microscopy. It was evaluated the exposure to nanoparticles of copper, calcium carbonate and welding smoke ultrafine particles. The methodology of qualitative evaluation, although requires some degree of conditioning, showed being a especially useful tool to define protection systems in nanoparticles case, whose toxicity is uncertain and we do not have established techniques of measuring them or permissible limits. Traditional measuring methods of industrial hygiene do not bring enough information to evaluate the exposure, and they must be complemented with electronic microscopy to characterize the size and chemical composition, key parameters in nanoparticles toxicity. This work provides a base of information and knowledge in a exploratory level, which is estimated as a useful tool to evaluate the exposure risk in applications that may appear in the current nanotechnology development in our country.

Key words: NANOTECHNOLOGY, NANOPARTICLES, NANOMATERIALS, INDUSTRIAL HYGIENE, QUALITATIVE EVALUATION, QUANTITATIVE EVALUATION, TOXICITY, ULTRAFINE PARTICLES.
Correspondencia / Correspondence:

Rómulo Zúñiga Rojas

Ramón Carnicer 163, Providencia, Santiago.

Tel.: 56 (2) 25157564.

e-mail: rzuniga@achs.cl

Recibido: 10 de Julio 2013 / Aceptado: 08 de Octubre 2013

\section{INTRODUCCIÓN}

Desde hace aproximadamente una década, la nanotecnología se está desarrollando en forma creciente, siendo calificada de "revolución tecnológica”. Para los próximos años, se espera la aparición de un gran número de productos y aplicaciones y, por consecuencia, de procesos industriales con trabajadores expuestos a 
nanopartículas, sustancias cuyos riesgos para la salud son actualmente desconocidos o inciertos. Esta preocupación ha motivado, principalmente en Estados Unidos y Europa, a organismos de consumidores, de medio ambiente y de salud ocupacional, a destinar importantes recursos al estudio de los efectos de las nanopartículas sobre la salud y el medio ambiente.

En el presente trabajo se explora el desarrollo de la nanotecnología en nuestro país, se revisan los últimos hallazgos sobre la toxicidad de las nanopartículas, se aplican metodologías cualitativas y cuantitativas para identificar y evaluar la exposición a nanopartículas en ambientes de trabajo y se entregan recomendaciones para minimizar los riesgos de la exposición ocupacional.

\section{METODOLOGÍA}

Dado que la Nanotecnología en nuestro país se encuentra en una etapa emergente, esta investigación tiene un carácter de tipo exploratorio y se realiza de acuerdo con las siguientes etapas:

- Revisión bibliográfica del estado del conocimiento respecto de los riesgos para la salud, desarrollo de productos y aplicaciones de los nanomateriales.

- Identificación de universidades, centros de investigación y empresas donde se utiliza nanotecnología, y procesos donde existe exposición a partículas ultrafinas.

- Evaluación cualitativa del riesgo de exposición en las empresas identificadas basada en la metodología NTP 877 sobre "Evaluación del riesgo por exposición a nanopartículas mediante el uso de metodologías simplificadas", descrita en referencia ${ }^{1}$.

- Evaluación cuantitativa de las concentraciones ambientales utilizando los métodos convencionales que establece la normativa internacional y nacional de higiene industrial ${ }^{2}$, complementándola con la técnica de microscopia electrónica TEM y energía dispersiva de rayos X (EDX). Como referencia se utilizan los límites establecidos para las partículas a escala micrométrica.

Los análisis gravimétricos y de absorción atómica de las muestras se realizaron en el Laboratorio de Higiene Industrial de la Asociación Chilena de Seguridad y los análisis de microscopia electrónica y energía dispersiva de rayos $\mathrm{X}$, en el laboratorio de Microscopia Electrónica de la Facultad de Ciencias Físicas y Matemáticas de la Universidad de Chile.

\section{RESULTADOS}

\section{Evaluaciones cualitativas}

Identificadas las empresas y las nanopartículas, y analizados los procesos, se procedió a emplear la metodología NTP 877 [1], para evaluar cualitativamente la exposición ocupacional a nanopartículas considera dos parámetros, uno denominado "severidad", que toma en cuenta sus características tóxicas, y otro "probabilidad", que es función de la forma en que se da la exposición del trabajador. Cada uno de estos parámetros se clasifica en 4 categorías que se combinan en 16 posibles resultados, los cuales dan lugar a 4 niveles de riesgos asociados a recomendaciones de control de la exposición, tal como se presenta a continuación en la Tabla 1.

En la Tabla 2 se presenta un resumen de las evaluaciones cualitativas realizadas en las empresas.

Los nanotubos de carbón son los únicos con "severidad" alta, en tanto el resto de las nanopartículas evaluadas quedaron en la categoría de
Tabla 1

Matriz de Recomendaciones según nivel de riesgo.

$\begin{array}{lcccc} & \begin{array}{c}\text { Extremadamente } \\ \text { Improbable (0-25) }\end{array} & \begin{array}{c}\text { Poco Probable } \\ (26-50)\end{array} & \begin{array}{c}\text { Probable } \\ (51-75)\end{array} & \begin{array}{c}\text { Muy Probable } \\ (76-100)\end{array} \\ \begin{array}{l}\text { Muy Alta } \\ (76-100)\end{array} & \text { R3 } & \text { R3 } & \text { R4 } & \text { R4 } \\ \text { 窟 } \begin{array}{l}\text { Alta } \\ (51-75)\end{array} & \text { R2 } & \text { R2 } & \text { R3 } & \text { R4 } \\ \begin{array}{l}\text { Media } \\ (26-50)\end{array} & \text { R1 } & \text { R1 } & \text { R2 } & \text { R3 } \\ \begin{array}{l}\text { Baja } \\ (0-25)\end{array} & \text { R1 } & \text { R1 } & \text { R1 } & \text { R2 }\end{array}$

R1: Ventilación General

R2: Ventilación por extracción localizada o campana laboratorio

R3: Confinamiento

R4: Buscar asesoramiento especializado

Ref. NTP 877, Evaluación del riesgo por exposición a nanopartículas mediante el uso de metodologías simplificadas, Instituto Nacional de Seguridad e Higiene en el Trabajo [1].

Tabla 2.

Resumen de Evaluaciones Cualitativas.

\begin{tabular}{|c|c|c|c|c|}
\hline $\begin{array}{l}\text { Empresa o } \\
\text { Institución/ } \\
\text { Actividad de } \\
\text { la Aplicación }\end{array}$ & Proceso & Severidad & Probabilidad & $\begin{array}{l}\text { Recomendación } \\
\text { Según Tabla } 1\end{array}$ \\
\hline $\begin{array}{l}\text { Empresa } 1 \\
\text { Laboratorio de } \\
\text { Investigación. }\end{array}$ & $\begin{array}{l}\text { Limpieza de evaporador } \\
\text { donde se producen } \\
\text { nanomateriales de } \mathrm{Cu} \text {. }\end{array}$ & $\begin{array}{l}\text { MEDIA } \\
42,0\end{array}$ & $\begin{array}{l}\text { Poco } \\
\text { Probable } \\
26,25\end{array}$ & $\begin{array}{l}\text { Ventilación } \\
\text { General }\end{array}$ \\
\hline $\begin{array}{l}\text { Empresa } 2 \\
\text { Centro } \\
\text { Odontológico. }\end{array}$ & $\begin{array}{l}\text { Uso de resina, en } \\
\text { restauración de piezas } \\
\text { dentales. La resina } \\
\text { contiene nanopartículas } \\
\text { de Sílice de } 20 \mathrm{~nm} \text {. }\end{array}$ & $\begin{array}{l}\text { MEDIA } \\
42,0\end{array}$ & $\begin{array}{l}\text { Poco } \\
\text { Probable } \\
26,25\end{array}$ & $\begin{array}{l}\text { Ventilación } \\
\text { General }\end{array}$ \\
\hline $\begin{array}{l}\text { Empresa } 3 \\
\text { Comercializadora } \\
\text { de productos con } \\
\text { nanopartículas }\end{array}$ & $\begin{array}{l}\text { Venta y almacenamiento } \\
\text { de productos de aseo e } \\
\text { impermeabilizantes, } \\
\text { solubles en agua, no } \\
\text { tóxicos. }\end{array}$ & $\begin{array}{l}\text { BAJA } \\
19,5\end{array}$ & $\begin{array}{l}\text { Extrema- } \\
\text { damente } \\
\text { Improbable } \\
13,75\end{array}$ & $\begin{array}{l}\text { Ventilación } \\
\text { General }\end{array}$ \\
\hline $\begin{array}{l}\text { Empresa } 4 \\
\text { Comercializadora } \\
\text { de productos con } \\
\text { nanopartículas. }\end{array}$ & $\begin{array}{l}\text { Aplicación de aditivo para } \\
\text { estabilizar suelos, producto } \\
\text { contiene nanopartículas } \\
\text { de fierro. } \\
\text { Aplicación de productos } \\
\text { para la remediación de } \\
\text { efluentes y suelos, } \\
\text { productos contienen } \\
\text { nanoparticulas de fierro. }\end{array}$ & $\begin{array}{l}\text { MEDIA } \\
27,5\end{array}$ & $\begin{array}{l}\text { Extrema- } \\
\text { damente } \\
\text { Improbable } \\
25 \\
\text { Extrema- } \\
\text { damente } \\
\text { Improbable } \\
25\end{array}$ & $\begin{array}{l}\text { Ventilación } \\
\text { General }\end{array}$ \\
\hline $\begin{array}{l}\text { Empresa } 5 \\
\text { Laboratorio de } \\
\text { Investigación. }\end{array}$ & $\begin{array}{l}\text { Recubrimiento de electrodos } \\
\text { (biosensores) con una } \\
\text { suspensión de nanotubos } \\
\text { de carbono. }\end{array}$ & $\begin{array}{l}\text { ALTA } \\
57,5\end{array}$ & $\begin{array}{l}\text { Poco } \\
\text { Probable } \\
43,75\end{array}$ & $\begin{array}{l}\text { Ventilación } \\
\text { Extracción } \\
\text { Localizada }\end{array}$ \\
\hline $\begin{array}{l}\text { Empresa } 6 \\
\text { Laboratorio de } \\
\text { Investigación. }\end{array}$ & $\begin{array}{l}\text { Producción de nanopar- } \\
\text { tículas de oro en solución } \\
\text { para diagnóstico y } \\
\text { tratamiento. }\end{array}$ & $\begin{array}{l}\text { BAJA } \\
20,0\end{array}$ & $\begin{array}{l}\text { Extrema- } \\
\text { damente } \\
\text { Improbable } \\
23,75\end{array}$ & $\begin{array}{l}\text { Ventilación } \\
\text { General }\end{array}$ \\
\hline
\end{tabular}

"severidad" media o baja. Por su parte, los procesos o formas en que se utilizan estos nanomateriales, calificaron la exposición como extremadamente improbable o poco probable. El único proceso que resultó con una recomendación de ventilación por extracción localizada fue el de recubrimiento de electrodos con nanotubos para mejoramiento de biosensores. Para el resto resultó suficiente la ventilación general.

\section{Evaluaciones cuantitativas}

A continuación se presentan los resultados obtenidos en la evaluación de tres procesos: Limpieza de equipo experimental de producción de nanopartículas de cobre, dosificación de nano carbonato de calcio en proceso de producción de pinturas y soldadura al arco de estructura de acero. 


\section{Limpieza de equipo experimental Antecedentes del Proceso}

El equipo corresponde a una cámara de evaporación a escala de laboratorio, utilizada para la producción de nanopartículas de un metal -en este caso cobre- sometido a un arco eléctrico, mediante evaporación al vacio, en atmósfera inerte. Las paredes de la cámara, soportes, partes de la bomba de vacío y diferentes mecanismos del sistema se ensucian y es necesario limpiarlos, actividad que es realizada por un investigador en forma manual, utilizando papel humedecido en alcohol (isopropílico o etílico) o acetona, tarea en la que demora alrededor de 30 minutos.

\section{Mediciones y Resultados}

Durante la limpieza del evaporador se tomaron simultáneamente dos muestras personales y dos muestras ambientales utilizando filtros de celulosa de $37 \mathrm{~mm}$ de diámetro. Tres de estos filtros, los dos personales y uno ambiental, se conectaron a bombas gravimétricas marca Gilian Modelo Gil Air 3, y uno, que corresponde a la muestra ambiental restante, fue instalado en un equipo marca MIE modelo RAM 2, el cual además de colectar la muestra de polvo en el filtro, permitió obtener la concentración en función del tiempo. Los caudales de aire movilizados por las bombas y el equipo RAM 2 se ajustaron en el orden de $2 \mathrm{~L} / \mathrm{min}$.

El objetivo de tomar las muestras personales y ambientales por duplicado, utilizando filtros de celulosa fue realizar primero el análisis gravimétrico de todas las muestras, para obtener las correspondientes concentraciones de polvo total en suspensión y, luego, utilizando los mismos filtros, analizar una muestra ambiental y una personal por la técnica de absorción atómica, de acuerdo con la metodología tradicional de la higiene industrial, y sus correspondientes duplicados por el método de microscopia de transmisión de electrones (TEM) y energía dispersiva de rayos $\mathrm{X}$ (EDX).

La cantidad total de polvo colectado en los filtros no fue suficiente para obtener por gravimetría valores confiables de las concentraciones de polvo, razón por la cual estos resultados no fueron informados. El filtro, en el que se colectó mayor cantidad de polvo, fue el de la muestra ambiental tomada con el equipo RAM, por lo cual fue seleccionado para analizar su contenido de cobre mediante absorción atómica, obteniéndose una masa de aproximadamente 0,5 microgramos, valor que corresponde a una concentración de $0,00855 \mathrm{mg} / \mathrm{m}^{3}$, resultando significativamente inferior al Límite Permisible Ponderado $\left(0,8 \mathrm{mg} / \mathrm{m}^{3}\right)$.

En la Figura 1 se presenta la variación de la concentración de polvo de $\mathrm{Cu}$ obtenida con el equipo RAM 2, el cual fue programado para registrar datos cada 1 minuto. Dado que el análisis gravimétrico no permitió obtener las concentraciones de polvo total colectado en los filtros no fue posible calibrar las lecturas que entrega el equipo en $\mathrm{mg} / \mathrm{m}^{3}$, por lo cual se optó por corregirlas con el criterio de que la concentración máxima fuera de orden de 100 para facilitar la comparación de los valores registrados.

Los primeros 8 minutos de medición correspondieron al polvo ambiental de fondo del laboratorio, el cual se encontró en el orden de un 17\% del valor máximo obtenido durante el periodo de medición; luego, cuando se inició la limpieza del equipo, se observaron sucesivos peaks de concentración, cuya tendencia se correlaciona bien con la emisión que corresponde a las distintas actividades realizadas, destacándose los peaks obtenidos cuando se limpió el soporte de la placa y el sector de la bomba de vacio, instantes en que se obtuvieron, respectivamente, máximos de concentración en el orden de 3 a 5 veces el valor de fondo.
Figura 1.

Variación de la concentración de polvo.

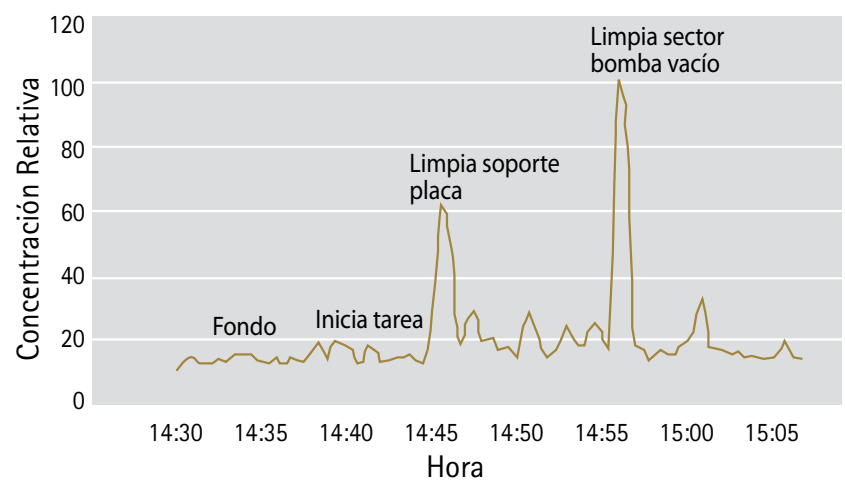

Figura 2.

Imágenes de las nanopartículas de cobre, $\mathrm{Cu}$, en el modo de campo claro y campo oscuro usando un HR/STEM.

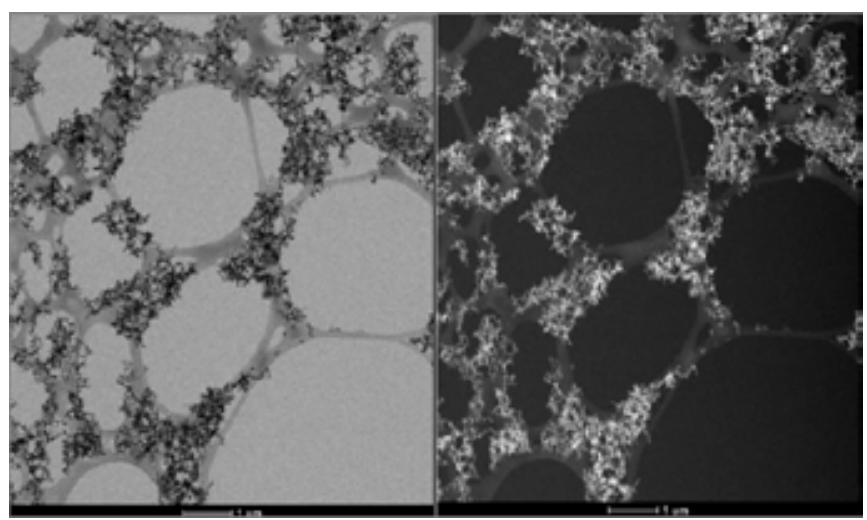

\section{Resultados Análisis TEM}

En la Figura 2 se presenta una imagen de las nanopartículas colectadas en una de las muestras ambientales.

Se observa que las partículas son de formas esféricas $\mathrm{u}$ ovaladas con un tamaño medio entre $20 \mathrm{~nm}$ y $40 \mathrm{~nm}$ y un grado menor de aglomeración; están unidas como "racimos" sin perder su individualidad o llegar a aglomerarse, formando cúmulos o "cluster". El análisis elemental obtenido por EDX (Figura 3), confirma que las nanopartículas son de cobre. El carbono que se detecta corresponde a la rejilla soporte utilizada en el TEM.

\section{Figura 3.}

Imagen de las nanopartículas de Cu mostrando la zona donde se le realizó análisis químico elemental y su correspondiente espectro de EDS.

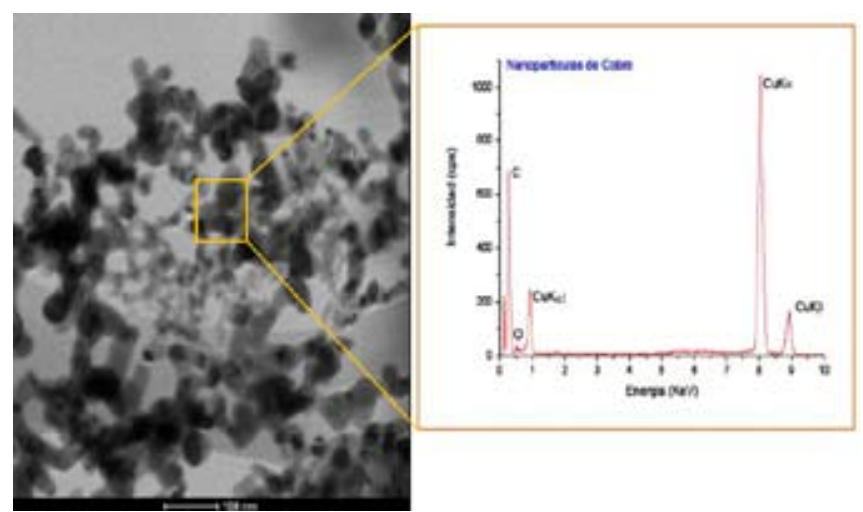


Si se considera que las partículas tienen un diámetro promedio del orden de $30 \mathrm{~nm}$, que la masa colectada en el filtro fue de 0,5 microgramos, que el volumen de aire que pasó por el filtro fue de 0,05845 $\mathrm{m}^{3}$ y que la densidad de cobre es de $9 \mathrm{~g} / \mathrm{cm}^{3}$, la concentración promedio de partículas durante la limpieza sería de 67x109 (partículas $/ \mathrm{m}^{3}$ ) que equivale a 67.000 (partículas $/ \mathrm{cm}^{3}$ ), valor que indica un nivel de exposición importante a diferencia de la señal que entrega la correspondiente concentración másica, cuyo valor de $0,00855 \mathrm{mg} /$ $\mathrm{m}^{3}$ representa una exposición muy inferior al límite permisible. Si la toxicidad de las partículas de cobre fuera mayor a escala nanométrica el valor de concentración medido en (partículas $/ \mathrm{cm}^{3}$ ) sería el parámetro correcto para evaluar la exposición; no obstante, como en el caso de este metal no existe evidencia científica que así lo demuestre, la concentración másica, que indica un nivel de riesgo aceptable, es el resultado que debería prevalecer. Además, esta posición se puede sustentar por el hecho de que los humos de cobre normalmente han contenido partículas ultrafinas y, por lo tanto, los estudios existentes sobre la toxicidad de este metal incluyen el efecto sobre la salud de los tamaños a escala nanométrica, lo cual probablemente ha influido para que el Límite Permisible Ponderado del Cobre en forma de humos sea casi 5 veces menor que en forma de polvo o nieblas [2].

\section{Dosificación de nanocarbonato de calcio Antecedentes del Proceso}

El carbonato de calcio se utiliza para mejorar el poder de cobertura de la pintura. La tarea, cuya exposición a polvo se evalúa, consiste en el pesaje de la cantidad necesaria para la preparación de un lote de pintura.

\section{Mediciones y Resultados}

El operador preparó 6 cargas, con pesos entre 2 a $11 \mathrm{Kg}$, demorando un total de 3,5 minutos. Durante este período, se tomaron dos muestras de polvo total, una ambiental, ubicada sobre la fuente, y una personal, colocada en la zona respiratoria del operador. Las muestras se tomaron utilizando filtros de PVC de 37 $\mathrm{mm}$ conectados a bombas gravimétricas marca Gilian Modelo Gil Air 3. En la Tabla 3 se presentan las concentraciones obtenidas. Los valores obtenidos fueron de 48,9 a $290,3 \mathrm{mg} / \mathrm{m}^{3}$ y superan en

Tabla 3.

Concentraciones de carbonato de calcio.

\begin{tabular}{lcc}
$\begin{array}{l}\text { Identificación } \\
\text { Muestra (N }\end{array}$ & $\begin{array}{c}\text { Tiempo muestreo } \\
\text { minutos }\end{array}$ & $\begin{array}{c}\text { Concentración } \\
\mathrm{mg} / \mathrm{m}^{3}\end{array}$ \\
\hline Ambiental (F4548) & 3,5 & 48,9 \\
Personal (F4549) & 3,5 & 290,3
\end{tabular}

más de 5 veces el límite permisible ponderado de $8 \mathrm{mg} / \mathrm{m}^{3}$, condición que no es aceptable de acuerdo al Artículo 60 del Decreto Supremo $\mathrm{N}^{\circ}$ 594/1999. Por otra parte, el valor de la muestra personal superó en, aproximadamente, 6 veces el valor de la muestra ambiental, lo que se explica debido al acercamiento que tiene el operador con el punto de emisión.

\section{Resultados Análisis TEM}

En la Figura 4 se presenta la imagen obtenida en microscopio electrónico de alta resolución de las partículas colectadas en la muestra ambiental (Filtro 4548). También se incluye el espectro obtenido por EDX para identificar la composición elemental de éstas.

Los tamaños encontrados son del orden de los $50 \mathrm{~nm}$, de formas aproximadamente ovaladas o esféricas y en el espectro se observa claramente el peak del Calcio, Ca, que identifica a las partículas de carbonato.
Figura 4.

Imagen y espectro EDS de la partículas colectadas en la Muestra Ambiental (Filtro 4548).
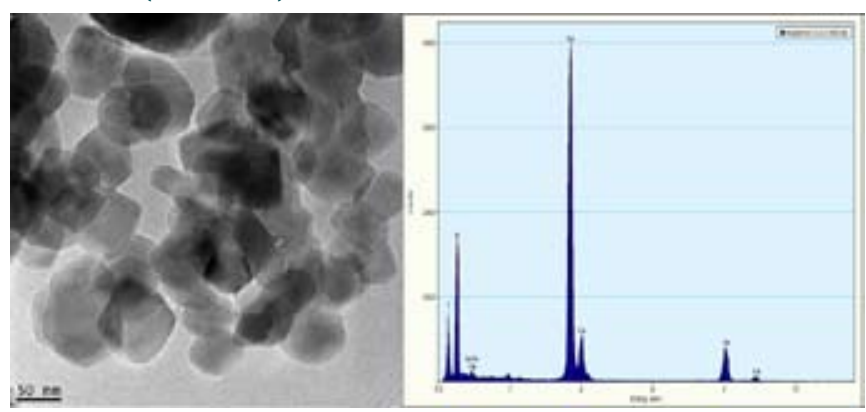

En la Figura 5 se presenta la imagen de una partícula con tamaño del orden de $50 \mathrm{~nm}$ micrones en cuyo espectro también se observa claramente el peak del calcio que la identifica como carbonato de calcio. La observación de esta partícula indica que en la muestra colectada existía también una cantidad importante de tamaños mayores a los nanométricos.

En la Figura 6 se muestra una imagen de las partículas colectadas en la muestra personal, Filtro 4549. En este caso, a diferencia de la muestra anterior, además de las partículas de nano carbonato, el análisis elemental de la muestra, EDS, indica también la presencia de nanopartículas de zinc, $\mathrm{Zn}$, las cuales probablemente provienen del proceso de mezcla de pigmentos que se realizaba en un sector contiguo.

\section{Soldadura al arco \\ Antecedentes del Proceso}

Se evalúa la exposición a humos en tarea de soldadura al arco de una estructura de acero ubicada al interior de un galpón. Los

\section{Figura 5.}

Imagen y espectro de partícula de tamaño micro de Muestra Ambiental (Filtro 4548).
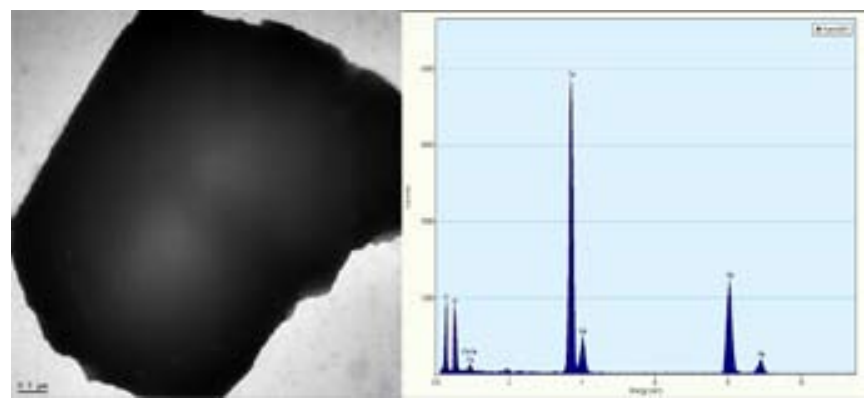

Figura 6.

Imagen TEM y Espectro EDS de Muestra Personal (Filtro 4549).

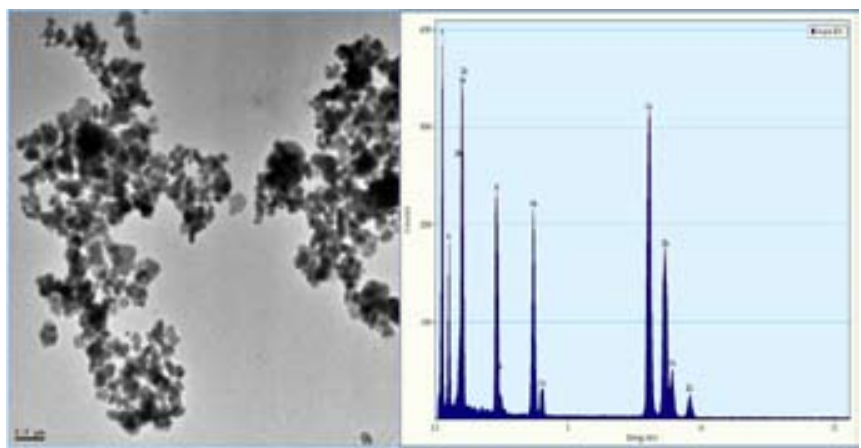


humos generados contienen, principalmente, Hierro (35-55\%), Manganeso (3-8\%) y Cobre (0,2\%), además de cantidades menores de Magnesio (0,1\%).

\section{Mediciones y Resultados}

Se tomaron muestras del humo emitido durante una tarea de soldadura que consumió 50 electrodos, utilizando filtros de celulosa de $37 \mathrm{~mm}$ conectados a bombas portátiles Gilian Modelo Gil Air 3. Estas incluyeron una muestra personal, instalando el filtro a la altura de las vías respiratorias del soldador y varias muestras ambientales en las cercanías del sector de soldadura para análisis mediante espectrofotometría de absorción atómica. Además, se tomó una muestra ambiental junto al soldador, Filtro 977, especial para análisis por microscopia electrónica.

En la Tabla 4 se presentan las concentraciones de metales obtenidas en el análisis por absorción atómica.

Tabla 4.

Concentracion de metales.

\begin{tabular}{lcc}
$\begin{array}{l}\text { Identificación } \\
\text { Muestra ( } \mathrm{N}^{\circ} \text { Filtro) }\end{array}$ & $\begin{array}{c}\text { Tiempo muestreo } \\
\text { minutos }\end{array}$ & $\begin{array}{c}\text { Concentración } \\
\mathrm{mg} / \mathrm{m}^{3}\end{array}$ \\
\hline $\begin{array}{l}\text { Personal (F980) } \\
\mathrm{Fe}\end{array}$ & & \\
$\mathrm{Mn}$ & 90 & 10,9 \\
$\mathrm{Cu}$ & & 1,2 \\
$\mathrm{Ambiental}(\mathrm{F975})$ & & $<1,02$ \\
$\mathrm{Fe}$ & 90 & 0,10 \\
$\mathrm{Mn}$ & & 0,02 \\
$\mathrm{Cu}$ & & $\mathrm{NSD}$ \\
\hline
\end{tabular}

Los resultados indican que los principales metales presentes en los humos corresponden a Fierro y Manganeso, con una baja presencia de Cobre, encontrándose las mayores concentraciones en la muestra personal del soldador, dada la cercanía que él tiene con el foco de emisión de humos. En este caso es importante señalar que para el Manganeso la concentración resultó igual a $1,2 \mathrm{mg} / \mathrm{m}^{3}$, valor que supera el Límite Permisible ponderado que establece el Decreto Supremo $\mathrm{N}^{\circ} 594 / 1999$.

\section{Resultados Análisis TEM}

En la Figura 7 se presenta una imagen de las partículas colectadas en el Filtro 977. Se observan partículas de tamaños muy diferentes, pero en general bajo los $200 \mathrm{~nm}$.

El análisis elemental por energía dispersiva de rayos X obtenido para el total de las partículas indica que están conformadas por $\mathrm{O}(\mathrm{K}), \mathrm{Na}(\mathrm{K})$, $\mathrm{Mg}(\mathrm{K}), \mathrm{Si}(\mathrm{K}), \mathrm{P}(\mathrm{K}), \mathrm{S}(\mathrm{K}), \mathrm{Cl}(\mathrm{K}), \mathrm{K}(\mathrm{K}), \mathrm{Mn}(\mathrm{K}), \mathrm{Ca}(\mathrm{K}), \mathrm{y} \mathrm{Fe}(\mathrm{K})$, como se aprecia en los espectros que acompañan las imágenes presentadas en la

Figura 7.

Imágenes HRTEM de las nanopartículas obtenidas en el filtro.

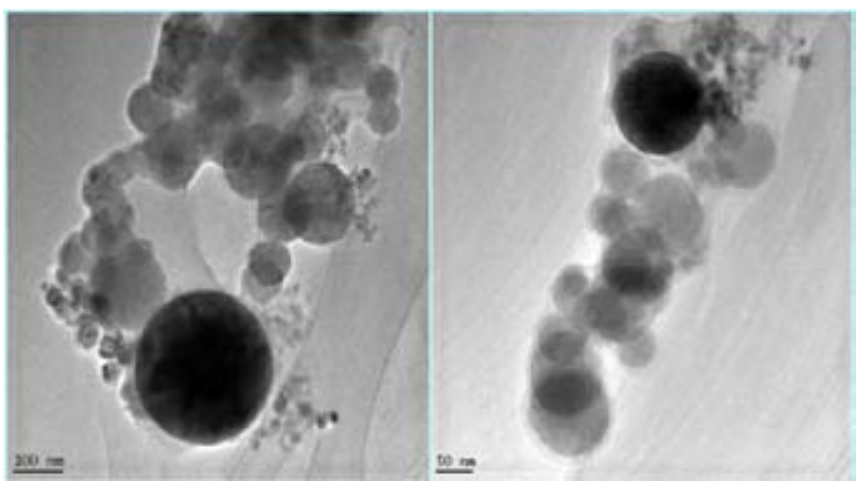

Figura 8.

Imágenes de HRTEM de las nanopartículas colectadas en Filtro 977, junto con su análisis elemental EDX.

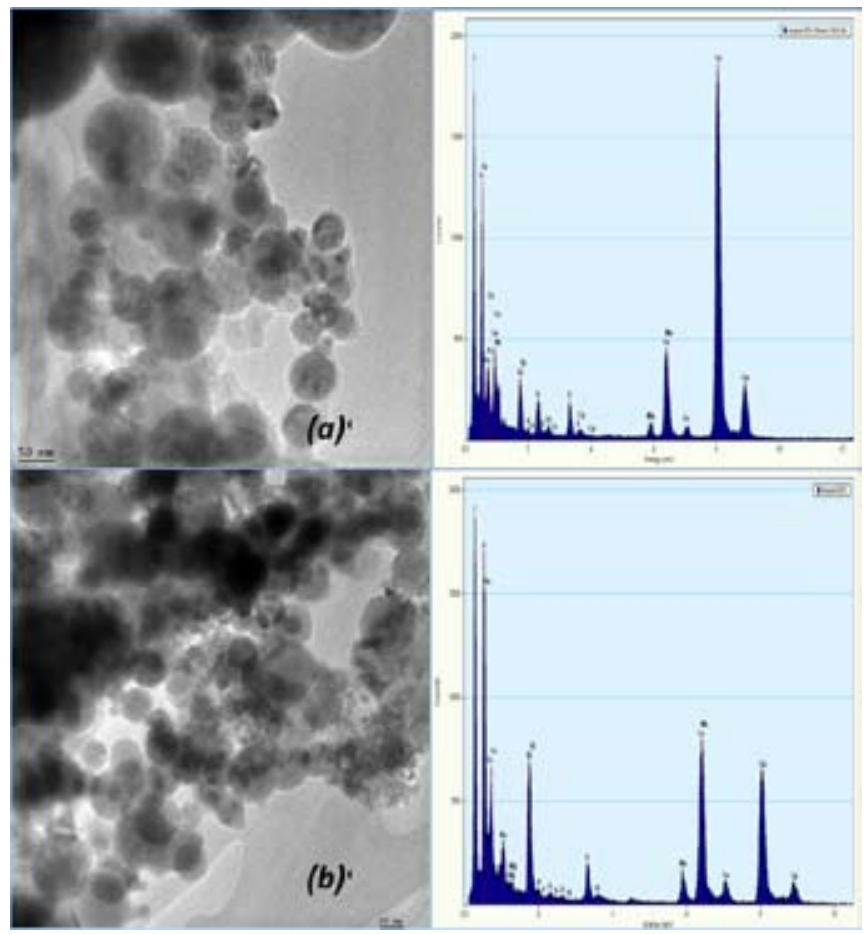

Figura 8. No se realizó este análisis para identificar en forma individual la composición de cada partícula, no obstante la experiencia indica que las partículas más pequeñas son las que corresponden a sílice.

En la Tabla 5 se presenta una cuantificación de los resultados del análisis elemental de las nanopartículas de la Figura 8, que incluye los porcentajes en peso atómico de cada elemento identificado, sin considerar el cobre porque corresponde a la rejilla porta-muestra y el carbono que se forma al hacer este análisis. La presencia mayoritaria de oxígeno es debido a que la mayoría de los compuestos formados son óxidos. El fierro, Fe, es el que le sigue en mayor cantidad, y después está el manganeso, Mn, lo que concuerda con el análisis por absorción atómica. Del resto de los compuestos identificados destaca, desde un punto de vista higiénico, la sílice, porque se encuentra en una proporción similar a la del manganeso y, aparentemente, formando parte de las partículas más pequeñas, las del orden de $20 \mathrm{~nm}$. Se debe considerar que la sílice, en este caso amorfa, normalmente no es considerada como un agente de riesgo que forme parte de los humos de soldadura.

Tabla 5.

Análisis Elemental de las nanopartículas en humos de soldadura.

$\begin{array}{lcccc}\begin{array}{l}\text { Elemento } \\ (\mathrm{K})\end{array} & \begin{array}{c}\text { Nanopartículas } \\ \text { Figura 8 (a) } \\ \text { \% peso }\end{array} & \begin{array}{c}\text { Nanopartículas } \\ \text { Figura 8 (a) } \\ \text { \% atómico }\end{array} & \begin{array}{c}\text { Nanopartículas } \\ \text { Figura 8 (b) } \\ \text { \% peso }\end{array} & \begin{array}{c}\text { Nanopartículas } \\ \text { Figura 8 (b) } \\ \text { \% atómico }\end{array} \\ \mathrm{O} & 45,89 & 67,42 & 44,12 & 67,65 \\ \mathrm{Na} & 5,74 & 5,47 & 3,40 & 3,63 \\ \mathrm{Mg} & 0,96 & 0,93 & 0,52 & 0,53 \\ \mathrm{Si} & 6,68 & 5,59 & 9,66 & 8,43 \\ \mathrm{P} & 0,17 & 0,13 & 0,66 & 0,52 \\ \mathrm{~S} & 4,72 & 3,46 & 0,27 & 0,21 \\ \mathrm{Cl} & 1,16 & 0,77 & 0,18 & 0,12 \\ \mathrm{~K} & 5,89 & 3,54 & 4,06 & 2,54 \\ \mathrm{Mn} & 0,75 & 0,44 & 5,64 & 2,52 \\ \mathrm{Ca} & 4,11 & 1,76 & ----- & ---- \\ \mathrm{Fe} & 23,88 & 10,05 & 31,44 & 13,81\end{array}$




\section{DISCUSIÓN}

Actualmente, se produce una gran variedad de nanopartículas, pero aún se puede considerar que su cantidad es reducida si se compara, por ejemplo, con la gran variedad de compuestos químicos que surgieron con el desarrollo de la industria petroquímica. Las nanopartículas producidas en mayor cantidad corresponden a nanoparticulas de metales, como la plata, el oro, el fierro, el platino, óxidos metálicos, como el dióxido de titanio, el óxido de fierro y el óxido de zinc, nanopartículas de silicio y las formadas por distintas estructuras de carbón, como los fulerenos (buckyballs), los grafenos y los nanotubos. Una característica importante de estos productos es que la mayor parte de ellos ya existían como subproducto de otros procesos industriales y, por lo tanto, ya hay antecedentes de la exposición laboral o ambiental; un ejemplo son las nanopartículas de óxidos metálicos, que forman parte de los humos metálicos, o las estructuras de carbón generadas en procesos de combustión como los humos emitidos por motores diésel.

En general, las nanopartículas se utilizan en proyectos de investigación, principalmente, en las áreas médicas, ambientales y en ciencias básicas. También se usan como materias primas para uso industrial y en productos de consumo, los cuales normalmente forman parte de una estructura que las mantiene encapsuladas, lo que minimiza o elimina el riesgo para los usuarios o consumidores. Si bien una institución como NIOSH mantiene un programa de evaluación de la exposición a nanopartículas en ambientes de trabajo, en la bibliografía revisada no se encontró información o datos sobre las concentraciones a las cuales se exponen los trabajadores en ambientes laborales donde se producen o utilizan nanopartículas; probablemente esto se deba a la falta de métodos aprobados de muestreo y análisis y a la carencia de límites o estándares que regulen la exposición.

La información existente a la fecha referenciada en la bibliografía revisada, respecto de la toxicidad de las nanopartículas permite concluir que las consecuencias de la exposición se agravan a escala nanométrica, como lo demuestran las experiencias en animales expuestos a dióxido de titanio ${ }^{3,5,6,7}$ y el conocimiento acumulado con la exposición humana a las partículas ultrafinas que forman parte de los humos diésel y los humos metálicos, cuyos límites permisibles reflejan esta mayor toxicidad ${ }^{8,9,10,11}$. La respuesta que aún se mantiene pendiente es sobre el carácter cancerígeno que podrían tener las nanopartículas producto de la mayor reactividad química y capacidad de penetración a nivel celular, que le confiere su tamaño.

Si bien se acepta que la exposición a un determinado agente químico conlleva una mayor gravedad cuando se encuentra en escala nanométrica, no se han establecido límites permisibles específicos para regularla, debido a la falta de conocimiento o de información concluyente, antecedentes que requieren de tiempo. Los intentos más directos por instaurar límites han sido realizados por NIOSH ${ }^{13,14,15}$, Bayer e IFA ${ }^{4,12}$; no obstante los valores recomendados no son consistentes. Por ejemplo, el límite de $0,1 \mathrm{mg} / \mathrm{m}^{3}$, que recomienda NIOSH para partículas de dióxido de titanio, tomando su densidad como $4,24 \mathrm{~g} / \mathrm{cm}^{3}$, corresponde al orden de 5.630 .481 (partículas $/ \mathrm{cm}^{3}$ ), en el caso de que el tamaño fuera $20 \mathrm{~nm}$, y del orden de 45.044 (partículas/ $\mathrm{cm}^{3}$ ) en el caso de que el tamaño fuera de $100 \mathrm{~nm}$, valores en general muy por encima del rango de 20.000 y 40.000 (partículas $/ \mathrm{cm}^{3}$ ) que recomienda IFA. Por otra parte, el límite de $0,05 \mathrm{mg} / \mathrm{m}^{3}$ que recomienda Bayer para nanotubos de carbón, suponiendo que tienen una densidad de $1,35 \mathrm{gr} / \mathrm{cm}^{3} \mathrm{y}$ largo de $150 \mathrm{~nm}$, equivale al orden de $785.950\left(\mathrm{NTC} / \mathrm{cm}^{3}\right)$, valor que no tiene relación con los límites permisibles de 0,16 (fibra/ $\mathrm{cm}^{3}$ ) y 1 fibra/cm³ que indica el Decreto Supremo N 594/1999 para el asbesto como crocidolita y para lana de vidrio, respectivamente. El problema principal que se tiene para medir la concentración de nanopartículas en aire es la necesidad de caracterizarlas por su tamaño y composición, para diferenciarlas del resto de las partículas, tanto las de tamaños mayores como las ultrafinas. En este sentido los métodos tradicionales presentan desventajas; la gravimetría no permite la distinción entre la masa de las distintas partículas colectadas en el filtro, lo que en principio se puede resolver si las partículas del proceso a evaluar están bien caracterizadas y se realiza una medida de la concentración de fondo del ambiente. No obstante, cuando las partículas son muy pequeñas y el proceso de emisión es de corta duración, como ocurrió en el caso de la limpieza de la cámara de producción de nanopartículas de cobre presentado en este trabajo, la masa colectada en el filtro es muy pequeña para ser cuantificada con precisión por gravimetría o absorción atómica. Una alternativa es la medición con equipos contadores de partícula como el RAM utilizado en este trabajo, pero se requiere una calibración del equipo para obtener resultados cuantitativos. Por el momento, para la etapa de evaluar los ambientes laborales en un contexto de investigación exploratoria, lo óptimo es siempre completar el análisis gravimétrico y de absorción atómica, con la caracterización del tamaño y composición de las partículas, mediante microscopia electrónica y energía dispersiva de rayos $\mathrm{X}$. De todas formas, se debe tener presente que se mantiene la incógnita de cuál es la masa neta de nanopartículas en las muestras.

La aceptación de que, a escala nanométrica, las partículas aumentan su peligrosidad y la incertidumbre existente sobre el carácter cancerígeno que éstas podrían tener hace aconsejable adoptar un criterio preventivo y limitar al máximo la exposición laboral mediante el uso de sistemas de ventilación y de protección personal, mientras no se establezcan límites permisibles y métodos aprobados para evaluar las concentraciones en aire. El método cualitativo utilizado en este trabajo es una herramienta que permite racionalizar la decisión de utilizar o no sistemas de control, pero insuficiente a juicio de los investigadores de este proyecto, para los procesos en los cuales se producen o manipulan nanopartículas, con aún incierta reactividad.

\section{CONCLUSIONES Y RECOMENDACIONES}

1. Actualmente, las nanopartículas de mayor aplicación son las producidas en base a metales, como la plata, el oro, el fierro y el platino, u óxidos metálicos, como el dióxido de titanio, el óxido de fierro y el óxido de zinc, y a estructuras de silicio y de carbón, como los fulerenos (buckyballs), los grafenos y los nanotubos.

Se recomienda, en particular, ser estricto en el control de la exposición de trabajadores a nanopartículas que correspondan a estructuras tipo fibras como ocurre con los nanotubos de carbón. En procesos que involucren la potencial dispersión de los nanoparticulas como mínimo se debe mantener la limpieza y orden en relación con la contaminación que éstas produzcan, manipular las nanopartículas utilizando sistemas de ventilación localizada como campanas de laboratorio o cajas de guantes que incluyan filtros para el control de las emisiones y utilizar elementos de protección personal, como guantes, lentes y ropa de uso exclusivo. 
2. La información existente a la fecha respecto de la toxicidad de las nanopartículas permite concluir que las consecuencias a la exposición son más graves cuando las partículas se presentan a escala nanométrica respecto de partículas en el rango de los micrones, siendo aún incierto que debido a la mayor reactividad química y capacidad de penetración a nivel celular que les confiere su reducido tamaño, puedan resultar cancerigenas.

Se recomienda adoptar un criterio preventivo, basado en el concepto de reducir la exposición del trabajador a nanopartículas utilizando lo más avanzado de la tecnología disponible.

3. No se han establecido límites permisibles específicos para regular la exposición a nanopartículas.

Para hacer evaluaciones cuantitativas, se recomienda considerar los siguientes criterios referenciales:

- En caso de existir límites para las partículas a escala micrométrica, tomar un 20\% de éste como límite de referencia para las nanoparticulas.

- Para el dióxido de titanio, tomar como límite de referencia el valor de $0,1 \mathrm{mg} / \mathrm{m}^{3}$ que propone NIOSH.

- Para nanopartículas de formas aproximadamente esféricas, considerar como referencia un límite entre 20.000 y 40.000 (partículas $/ \mathrm{cm}^{3}$ ), según si la densidad es mayor o menor a $6 \mathrm{~g} /$ $\mathrm{cm}^{3}$, respectivamente.

- Para estructuras tipo fibra, considerar como referencia un límite de 1 (fibra/ $\mathrm{cm}^{3}$ ), valor representativo de los límites que existen actualmente en nuestra legislación para los agentes químicos que se presentan en forma de fibras.

4. En Chile la nanotecnología está en una etapa incipiente, estando su principal desarrollo en los centros de investigación de las universidades, lo que indica que actualmente los investigadores y estudiantes son el principal grupo de personas expuestas a nanopartículas.

Se recomienda evaluar y controlar la exposición a nanopartículas de los investigadores de centros universitarios dedicados a la nanotecnología.

5. Los métodos tradicionales de la Higiene Industrial no se pueden aplicar en forma directa para evaluar la concentración de nanopartículas en aire, ya que es necesario caracterizarlas por su tamaño y composición, para diferenciarlas del resto de las partículas, tanto las de tamaño mayor como las ultrafinas. La microscopia TEM y la energía dispersiva de rayos $\mathrm{X}$ son técnicas que se pueden utilizar para hacer esto último.

Se recomienda realizar otros estudios y experiencias de evaluación que permitan validar la consistencia de los resultados obtenidos por la gravimetría tradicional, la espectrometría de absorción atómica, la microscopia TEM y la difracción de rayos X en muestras de aire tomadas en ambientes de trabajo. Es necesario desarrollar una metodología que, además de caracterizar las nanopartículas por su tamaño y composición, entregue el porcentaje o fracción que éstas representan del total de partículas colectadas en el filtro.

6. El análisis de la composición de las partículas ultrafinas en humos de soldadura identificó al silicio, probablemente en forma de sílice amorfa y tamaños del orden de $20 \mathrm{~nm}$, entre los elementos de mayor concentración colectados en el filtro. Se destaca este hallazgo porque normalmente la sílice no se considera entre los contaminantes que forman parte de los humos de soldadura.

Se recomienda realizar nuevos análisis de microscopia electrónica $\mathrm{y}$ energía dispersiva de rayos $\mathrm{X}$ a filtros de muestras de humos de soldadura para identificar, en particular, las nanopartículas con sílice y caracterizarlas de acuerdo a su tamaño, composición molecular, estructura cristalográfica y concentración másica. Se recomienda, además, complementar estos análisis, si corresponde, con el análisis tradicional que se le hace a las muestras de sílice, para determinar si estas partículas son o no de importancia higiénica.

\section{REFERENCIAS}

1. http://www.insht.es/InshtWeb/Contenidos/Documentacion/FichasTecnicas/ NTP/Ficheros/821a921/877w.pdf (visitada el 24-06-2013).

2. www.cdc.gov/niosh/docs/2003-154/method-c.html (visitada el 22-12-2012).

3. 2012 TLVs and BEls Documentation of the Threshold Limit Values for Chemical Substances and Physical Agents \& Biological Exposures Indices. ACGIH.

4. http://www.dguv.de/ifa/en/fac/nanopartikel/beurteilungsmassstaebe/index. jsp (visitada el 29 -12-2012).

5. G Oberdörster, J Ferin, R Gelein, S C Soderholm, and J Finkelstein; Role of the alveolar macrophage in lung injury: studies with ultrafine particles. Environ Health Perspect. 1992 July; 97: 193-199.

6. Warheit DB, Webb TR, Reed KL, Frerichs S, Sayes CM.B; Pulmonary toxicity study in rats with three forms of ultrafine-TiO2 particles: differential responses related to surface properties. Toxicology. 2007 Jan 25;230(1):90-104

7. http://monographs.iarc.fr/ENG/Monographs/PDFs/93-titaniumdioxide.pdf (Visitada el 18-11- 2012).

8. Lam CW, James TL, McCluskey R, Hunter RL.; Pulmonary toxicity of singlewall carbon nanotubes in mice 7 and 90 days after intratracheal instillation. Toxicol Sci. 2004 Jan;77(1):126-34. Epub 2003 Sep 26.

9. Oberdorster G, Gelein RM, Ferin J, Weiss B. Association of particulate air pollution and acute mortality:involvement of ultrafine particles?. Inhal Toxicol. 1995 Jan-Feb;7(1):111-24.

10. Debra T. Silverman et al. The Diesel Exhaust in Miners Study: A Nested CaseControl Study of Lung Cancer and Diesel Exhaust J Natl Cancer Inst. 2012 June 6; 104(11): 855-868.

11. http://www.irsst.qc.ca/media/documents/PublRSST/R-589.pdf (Visitada 30-11-12). 12.http://www.baytubes.com/news_and_services/news_091126_oel.html (Visitada el 23-11-2012).

13. Evaluation of Health Hazard and Recommendations for Occupational Exposure to Titanium Dioxide. NIOSH CURRENT INTELLIGENCE BULLETIN. November 2005.

14. NIOSH Nanotechnology Field Research Effort Fact Sheet. DHHS (NIOSH) Publication No. 2008-121. February 2008.

15.Approach to safe Nanotechnology.DHHS(NIOSH) Publication No. 2009-125. March 2009.

Referencias complementarias:

1. Project on Emerging Nanotechnologies. An inventory of nanotechnologybased consumer products currently on the market. http://www.nanotechproject.org/inventories/consumer/ (visitada 16-11-2012).

2. http://www.nanowerk.com/index.php (Visitada el 26 de diciembre 2012).

3. U.S. Department of Energy Nanoscale SCience Research Centers. Approach to Nanomaterial ES\&H. Revision 3a, May 12, 2008.

4. www.oecd.org/env/nanosafety (Visitada 19-11- 2012).

5. Ramachandran G. Et al. A Strategy for Assessing Workplace Exposures to Nanomaterials. Journal of Ocuppational and Environmental Hygiene, 8:673-685 Nov. 2011.

6. 2011 U.S. Intellectual Property Enforcement Coordinator Annual Report on Intellectual Property Enforcement Cover Title Here Report to the President and Congress on the Fourth Assessment of the National Nanotechnology Initiative. Executive Office of the President President's Council of Advisors on Science and Technology APRIL 2012. 